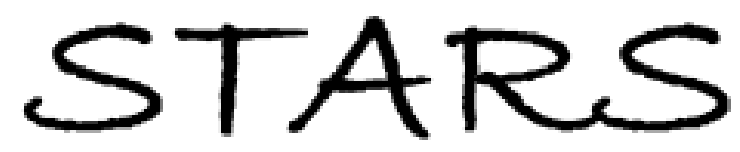

University of Central Florida

STARS

$1-1-2010$

\title{
Crystal coherence length effects on the infrared optical response of $\mathrm{MgO}$ thin films
}

\author{
J. F. Ihlefeld \\ J. C. Ginn \\ D. J. Shelton \\ University of Central Florida \\ V. Matias
}

M. A. Rodriguez

See next page for additional authors

Find similar works at: https://stars.library.ucf.edu/facultybib2010

University of Central Florida Libraries http://library.ucf.edu

This Article is brought to you for free and open access by the Faculty Bibliography at STARS. It has been accepted for inclusion in Faculty Bibliography 2010 s by an authorized administrator of STARS. For more information, please contactSTARS@ucf.edu.

\section{Recommended Citation}

Ihlefeld, J. F.; Ginn, J. C.; Shelton, D. J.; Matias, V.; Rodriguez, M. A.; Kotula, P. G.; Carroll, J. F. III; Boreman, G. D.; Clem, P. G.; and Sinclair, M. B., "Crystal coherence length effects on the infrared optical response of MgO thin films" (2010). Faculty Bibliography 2010s. 286.

https://stars.library.ucf.edu/facultybib2010/286

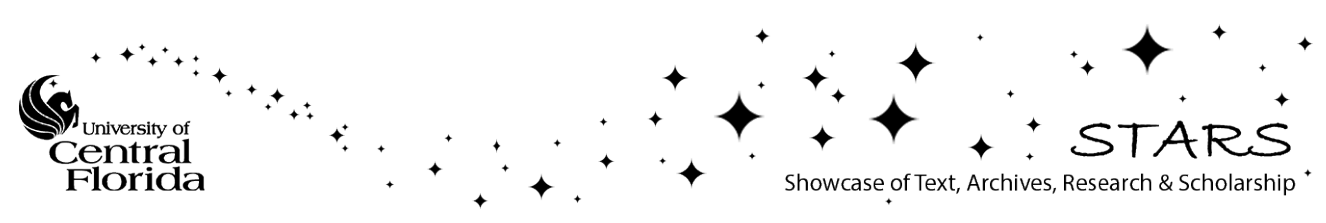




\section{Authors}

J. F. Ihlefeld, J. C. Ginn, D. J. Shelton, V. Matias, M. A. Rodriguez, P. G. Kotula, J. F. Carroll III, G. D. Boreman, P. G. Clem, and M. B. Sinclair 


\section{Crystal coherence length effects on the infrared optical response of $\mathrm{MgO}$ thin films}

Cite as: Appl. Phys. Lett. 97, 191913 (2010); https://doi.org/10.1063/1.3515901

Submitted: 29 September 2010 . Accepted: 23 October 2010 . Published Online: 12 November 2010

J. F. Ihlefeld, J. C. Ginn, D. J. Shelton, V. Matias, M. A. Rodriguez, P. G. Kotula, J. F. Carroll, G. D. Boreman, P. G. Clem, and M. B. Sinclair

\section{ARTICLES YOU MAY BE INTERESTED IN}

Recombination and loss analysis in polythiophene based bulk heterojunction photodetectors

Applied Physics Letters 81, 3885 (2002); https://doi.org/10.1063/1.1521244

Enhanced dielectric and piezoelectric responses in $\mathrm{Zn}_{1-x} \mathrm{Mg}_{\mathrm{x}} \mathrm{O}$ thin films near the phase separation boundary

Applied Physics Letters 110, 042903 (2017); https://doi.org/10.1063/1.4973756

Thermal conductivity of nano-grained $\mathrm{SrTiO}_{3}$ thin films

Applied Physics Letters 101, 231908 (2012); https://doi.org/10.1063/1.4769448

\section{Applied Physics Reviews} Now accepting original research 


\title{
Crystal coherence length effects on the infrared optical response of MgO thin films
}

\author{
J. F. Ihlefeld, ${ }^{1, a)}$ J. C. Ginn, ${ }^{1}$ D. J. Shelton, ${ }^{2}$ V. Matias, ${ }^{3}$ M. A. Rodriguez, ${ }^{1}$ P. G. Kotula, ${ }^{1}$ \\ J. F. Carroll III, ${ }^{1}$ G. D. Boreman, ${ }^{2}$ P. G. Clem, ${ }^{1}$ and M. B. Sinclair ${ }^{1}$ \\ ${ }_{1}^{1}$ Sandia National Laboratories, Albuquerque, New Mexico 87185, USA \\ ${ }^{2}$ College of Optics and Photonics/CREOL, University of Central Florida, Orlando, Florida 32816, USA \\ ${ }^{3}$ Los Alamos National Laboratory, Los Alamos, New Mexico 87545, USA
}

(Received 29 September 2010; accepted 23 October 2010; published online 12 November 2010)

\begin{abstract}
The role of crystal coherence length on the infrared optical response of $\mathrm{MgO}$ thin films was investigated with regard to Reststrahlen band photon-phonon coupling. Preferentially (001)-oriented sputtered and evaporated ion-beam assisted deposited thin films were prepared on silicon and annealed to vary film microstructure. Film crystalline coherence was characterized by $\mathrm{x}$-ray diffraction line broadening and transmission electron microscopy. The infrared dielectric response revealed a strong dependence of dielectric resonance magnitude on crystalline coherence. Shifts to lower transverse optical phonon frequencies were observed with increased crystalline coherence. Increased optical phonon damping is attributed to increasing granularity and intergrain misorientation. () 2010 American Institute of Physics. [doi:10.1063/1.3515901]
\end{abstract}

The study of optical devices built with low-loss negative-permittivity and permittivity-near-zero components is generating great interest, particularly for metamaterial applications. ${ }^{1,2}$ To implement materials into designs utilizing these phenomena, knowledge of the effect of defects and microstructure on optical response is necessary; this is especially true as device dimensions approach typical defect sizes on the order of nanometers. The coupling of infrared (IR) photons with near-zero wavevector optical phonons results in a band of high-reflectivity, commonly denoted the Reststrahlen band, in partially ionic materials. In this band the real portion of permittivity crosses zero and can become strongly negative. As the transverse optical phonon oscillations responsible for this response are collective phenomena requiring a degree of lattice coherence, knowledge of the role of crystal coherence length on the photon-phonon interactions is pertinent. Surface asperities, bulk porosity, and particle size are known to affect the spectral shape and intensity of the infrared optical response of ionic crystals, ceramics, and powders. ${ }^{3-9}$ These correlations, however, have been predominantly investigated for relatively large-scale micronsize features. The role of submicron defects including dislocations and crystallographic disorder are well known to affect phonon lifetimes and scattering (see, for example, Ref. 10 and citations within) but have been minimally studied in the context of the Reststrahlen band response in thin films. ${ }^{11}$

Magnesium oxide $(\mathrm{MgO})$ crystallizes in the rocksalt structure $(F m 3 m)$ and, owing to its high symmetry and two ion basis, possesses relatively simple phonon dispersion and Reststrahlen spectra. The Reststrahlen band in $\mathrm{MgO}$ occurs for incident optical wavelengths between $13.6 \mu \mathrm{m}(0.091$ $\mathrm{eV})$ and $25 \mu \mathrm{m}(0.049 \mathrm{eV}) .^{12}$ The effect of defects on phonon damping and IR optical response has been evaluated for ion implanted $\mathrm{MgO}$ single crystals previously. ${ }^{13}$ In this report we present the effect of crystal coherence length on the IR optical response of dense polycrystalline $\mathrm{MgO}$ thin films with equivalent thickness and surface roughness.
$200 \mathrm{~nm}$ thick $\mathrm{MgO}$ thin films were prepared on (001)oriented silicon substrates via $30^{\circ}$ off-axis radio frequency (rf) magnetron sputtering and ion-beam assisted deposition (IBAD). The substrates were subjected to a buffered HF etch and de-ionized- $\mathrm{H}_{2} \mathrm{O}$ rinse prior to loading into the loadlocked sputter chamber to remove the native $\mathrm{SiO}_{2}$ surface. A polycrystalline crystal coherence length series was prepared from films deposited by $\mathrm{RF}$ sputtering $\mathrm{MgO}$ from a singlephase sintered target in 5 mTorr of argon at room temperature with a sputter power density of $3.7 \mathrm{~W} / \mathrm{cm}^{2}$. The wafer was subsequently divided and sections were processed at $200-800{ }^{\circ} \mathrm{C}$ in $200{ }^{\circ} \mathrm{C}$ intervals in air for $1 \mathrm{~h}$. Thermodynamic calculations predict phase stability of $\mathrm{MgO}$ with silicon in this temperature range with no chemical reactions, suggesting that abrupt interfaces with no secondary phases should form in this process. ${ }^{14}$ A $200 \mathrm{~nm}$ thick biaxially textured (001)-oriented film was prepared via the IBAD method as described elsewhere. ${ }^{15}$ Briefly, a $5 \mathrm{~nm}$ amorphous $\mathrm{Y}_{2} \mathrm{O}_{3}$ layer is deposited by evaporation followed by a $\sim 5 \mathrm{~nm}$ IBAD-MgO layer. A $200 \mathrm{~nm}$ thick homoepitaxial layer is then deposited at $600{ }^{\circ} \mathrm{C}$ by electron-beam sublimation from a $\mathrm{MgO}$ source. Film roughness was assessed with atomic force microscopy (AFM). Crystal coherence length was characterized with x-ray diffraction (XRD) in both BraggBrentano (Philips MRD) and grazing-incidence (Siemens D-500 with $2^{\circ}$ alpha angle) geometries and transmission electron microscopy (TEM) (FEI Tecnai F30-ST) in TEM and scanning TEM modes. Crystal coherence lengths were determined using x-ray line broadening and Scherrer's formula. ${ }^{16}$ The structure factor for $\mathrm{MgO}$ limits the number of diffraction peaks and therefore precluded the use of the more rigorous Williamson-Hall ${ }^{17}$ method and subsequent decoupling of strain and coherence. Infrared optical properties were measured using an infrared variable angle spectroscopic ellipsometer (Woollam IR-VASE) at $50^{\circ}, 55^{\circ}$, and $60^{\circ}$ incidence angles for all samples over the range of 1.5-35 $\mu \mathrm{m}$. A least-squares fit for a Lorentzian resonator at the transverse optic phonon mode wavelength and a weak Gaussian fit in the mid-IR was performed to extract the com-

${ }^{\text {a)} E l e c t r o n i c ~ m a i l: ~ j i h l e f e @ s a n d i a . g o v . ~}$ 


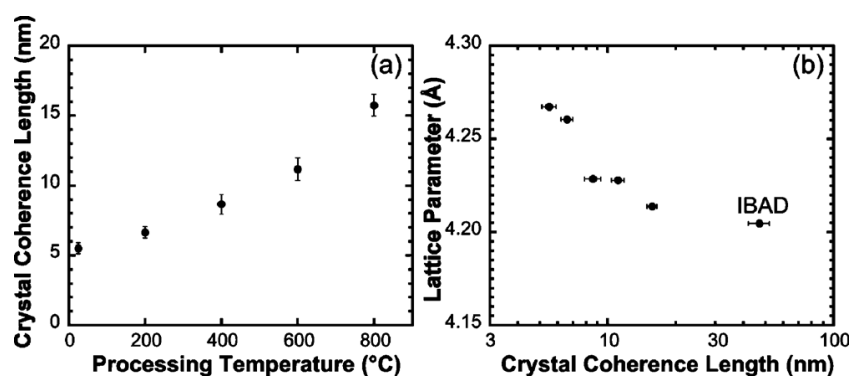

FIG. 1. (a) Crystal coherence length calculated from XRD FWHM of the 200-reflection of sputtered $\mathrm{MgO}$ films as-deposited and postprocessed in air from $200{ }^{\circ} \mathrm{C}$ to $800{ }^{\circ} \mathrm{C}$. (b) Lattice parameter calculated from the 200 reflection of $\mathrm{MgO}$ as a function of crystal coherence length.

plex refractive index components from the ellipsometer parameters. The effect of crystal coherence length on the optical response was quantified by fitting the imaginary component of permittivity calculated from the measured optical response with complex permittivity values derived from a classical oscillator model given in Eq. (1), ${ }^{18}$

$$
\varepsilon(\omega)=\varepsilon_{\infty}+\frac{\varepsilon_{0}-\varepsilon_{\infty}}{\left[1+\left(\frac{\omega^{2}}{\omega_{\mathrm{TO}}^{2}}\right)\right]-i\left(\frac{\omega \gamma}{\omega_{\mathrm{TO}}^{2}}\right)},
$$

where $\varepsilon_{0}, \varepsilon_{\infty}, \gamma, \omega$, and $\omega_{\mathrm{TO}}$ are the low frequency permittivity, high frequency permittivity, damping constant, incident photon frequency, and transverse optical phonon frequency, respectively. Values of $9.86,2.95$, and $11.8 \mathrm{THz}$ were used for the low frequency permittivity, high frequency permittivity, and transverse optic phonon frequency, respectively, ${ }^{19}$ as starting points and fits were introduced to determine damping constants and phonon frequencies.

Figure 1(a) shows a plot of the coherent scattering lengths for the polycrystalline $\mathrm{MgO}$ samples processed from room temperature to $800{ }^{\circ} \mathrm{C}$ and reveals a trend of increasing length with processing temperature. Measured coherent scattering lengths ranged from $5.5 \mathrm{~nm}$ (as-deposited sample) to $15.8 \mathrm{~nm}\left(800{ }^{\circ} \mathrm{C}\right.$ processed sample). Identical scattering lengths were calculated using the $200 \mathrm{MgO}$ x-ray reflection for both grazing-incidence and Bragg-Brentano diffraction geometries, suggesting that there is minimal directional dependence of coherent scattering lengths and that the features contributing to scattering are equiaxed. A coherent scattering length of $47 \mathrm{~nm}$ was measured for the (001)-oriented IBAD film (note that this is the minimum possible scattering length value as strain contributions could not be decoupled). Additionally, an omega rocking curve full width at half maximum (FWHM) value of $1.2^{\circ}$ for the 200-reflection and $4.7^{\circ}$ FWHM for the 220 reflection in phi were measured. $\mathrm{MgO}$ lattice parameters were calculated from the 200 peak position using the silicon 400 peak as a reference for sample displacement error correction ${ }^{20}$ with the results shown in Fig. 1(b). A systematic reduction in lattice parameter with crystalline coherence increase is observed. These data indicate that the higher the degree of crystalline perfection, the more closely the lattice parameter approaches the accepted single crystal value of $4.21 \AA$. It is likely that the grain boundary disorder in the fine crystallite size samples results in expanded lattice parameters, as has been suggested previously for fine-grained $\mathrm{MgO}{ }^{9}$
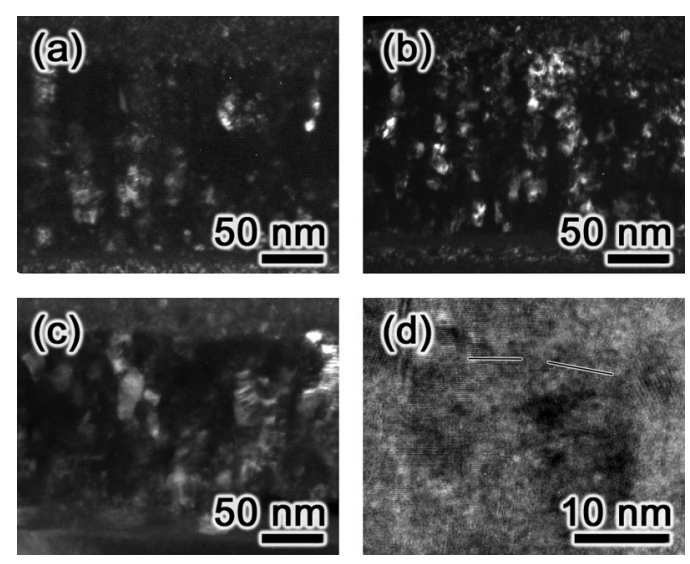

FIG. 2. Dark-field TEM images from (a) as-deposited, (b) $400{ }^{\circ} \mathrm{C}$ processed, and (c) $800{ }^{\circ} \mathrm{C}$ processed sputtered $\mathrm{MgO}$ thin films. (d) Highresolution TEM image of an as-deposited $\mathrm{MgO}$ film. Lines highlight (200) type planes for two adjacent grains.

TEM analysis of the thermally processed sputtered samples revealed that the samples were comprised of fine crystallites within larger columns consisting of preferentially crystallographic texturing with (001)-orientation. Dark-field images in Fig. 2 show bright regions where the beam satisfies diffraction conditions for individual crystallites in the as-deposited [Fig. 2(a)], $400{ }^{\circ} \mathrm{C}$ processed [Fig. 2(b)], and $800{ }^{\circ} \mathrm{C}$ processed [Fig. 2(c)] samples. Qualitatively, the average sizes of the observed individual crystallites agree well with the coherent scattering lengths measured via XRD. High-resolution images revealed small angle grain boundaries $\left(\sim 1^{\circ}-2^{\circ}\right)$ within the columnar textured regions with domain sizes nearly identical to the coherent $\mathrm{x}$-ray scattering length [Fig. 2(d)]. Lines highlighting two adjacent (002)-type planes have been drawn showing small angle tilts separating adjacent crystallites. Between larger (001)-oriented columns fine-scale porosity was observed, however no obvious change in density or porosity distribution was observed with annealing condition. Additionally, no change in thickness could be identified, suggesting that film density remained constant. No difference in film roughness was observed for the sputtered and thermally processed samples. The rms roughness values measured by AFM over $9 \mu \mathrm{m}^{2}$ were $6.86 \mathrm{~nm}+/-0.26 \mathrm{~nm}$.

The real and imaginary components of the optical permittivity between 2 and $35 \mu \mathrm{m}$ wavelengths are shown in Fig. 3 for the polycrystalline samples that were as-deposited and those processed at 400 and $800{ }^{\circ} \mathrm{C}$ in addition to the (001)-oriented IBAD-deposited sample. A single resonance
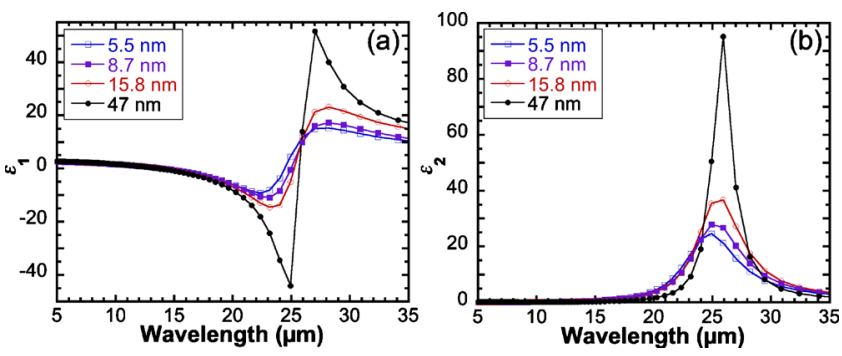

FIG. 3. (Color online) (a) Real and (b) imaginary components of the dielectric spectra obtained from infrared-spectroscopic ellipsometry analysis of thermally processed polycrystalline $200 \mathrm{~nm}$ thick $\mathrm{MgO}$ films and a $200 \mathrm{~nm}$ thick (001)-oriented biaxially textured IBAD film. 


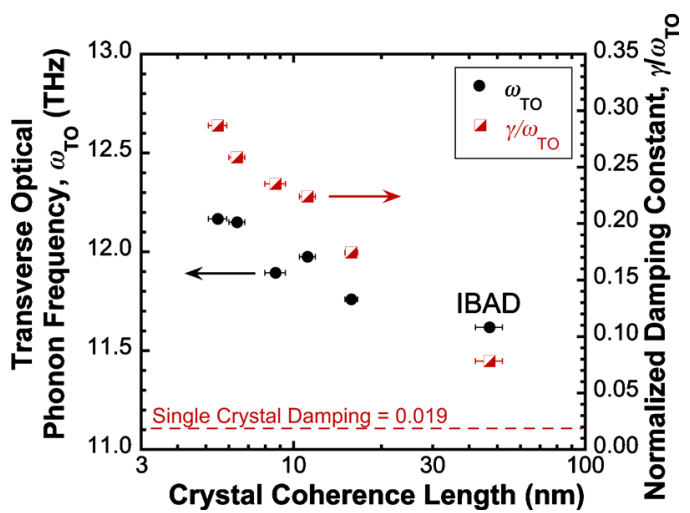

FIG. 4. (Color online) Transverse optical phonon frequency (closed circles) and normalized damping constant (open squares) for $200 \mathrm{~nm}$ thick $\mathrm{MgO}$ films as a function of crystal coherence length.

located at approximately $25 \mu \mathrm{m}$ is observed with no evidence of additional features that could be attributed to surface modes, as are commonly observed in fine-grained powder samples of similar dimensions at shorter wavelengths than the transverse optic mode. ${ }^{3,9}$ The magnitudes of the polarization resonance $\left(\varepsilon_{1}\right)$ and absorption $\left(\varepsilon_{2}\right)$ scale with coherent crystal length, as is evident in comparing the thermally processed samples with the IBAD sample. An increase in peak resonance amplitude by greater than $4.5 \times$ is observed between samples with $5.5 \mathrm{~nm}\left(\varepsilon_{1}=-9.1\right)$ and $47 \mathrm{~nm}$ $\left(\varepsilon_{1}=-44\right)$ crystalline coherence lengths. Additionally, a shift in the transverse optic phonon mode toward longer wavelengths (lower frequency) is observed as the crystal coherence length increases. Figure 4 shows the transverse optical phonon frequency and damping constant normalized to the transverse optical phonon frequency as a function of the coherent crystal length. Similar to the lattice parameter trend, the oscillation frequency also scales with crystallinity. This was observed previously in fine-grained $\mathrm{MgO}$ and the increased frequency with finer crystal size was attributed to the increased lattice parameter. ${ }^{9}$ An increased damping constant with decreased crystal coherence is observed; this appears to be consistent with optical phonon scattering at the defects responsible for finite crystal coherence lengths. As the textured column width and porosity levels were constant for each processing condition, this suggests that the damping constants are strongly influenced by the defects responsible for crystal coherence length limitations, e.g., grain boundaries, small angle tilt boundaries, and dislocations. It is prudent to mention that an apparent shift in the longitudinal optic mode (where $\varepsilon_{1}$ crosses zero) to shorter wavelengths was observed as crystalline coherence length increased. Combined with the transverse optic mode shift to longer wavelengths, this would indicate that the static and high frequency dielectric constants must also scale with crystallinity through the Lyddane-Sachs-Teller relation. Unfortunately, limited instrument resolution at long wavelengths limits our ability to rigorously assess the static dielectric constant and explore this effect further.

A line indicating the accepted room temperature single crystal normalized damping constant has been plotted in Fig. 4. It is evident that as the crystal coherence length increases, bulklike values for the normalized damping constant (0.019) are approached. ${ }^{21}$ It is worth noting that this suggests that even the high crystalline quality of the IBAD film is not sufficient for a bulk damping constant; substantial film thickness and lateral dimensions of high crystalline quality may be necessary to obtain a sharp resonance and subsequent high-reflectivity characteristic of the single crystalline Reststrahlen response, as has been demonstrated in other thin film systems. ${ }^{22}$ This may have implications on the feature size in both thickness and lateral dimensions for devices designed around the Reststrahlen response.

In summary, this study demonstrates that the Reststrahlen band response in $\mathrm{MgO}$ thin films strongly depends on the crystalline quality of the material. Films prepared with fine-scale crystallinity possess higher frequency transverse optical phonon modes and increased damping constants. The damping constant scales inversely with crystallinity resulting in an increased transverse optic phonon resonance with incident light. The reduced optical response caused by nanoscale defects may have beneficial implications for designing epsilon near-zero infrared materials, due to the flattened response through defect scattering, and may have broad implications for accurate design of IR optical components utilizing optical phonon modes in thin film materials.

The authors wish to acknowledge experimental advice and critical review from M. Lee, P. Rakich, and G. L. Brennecka. This research was supported by the Laboratory Directed Research and Development Program at Sandia National Laboratories. This work was performed, in part, at the Center for Integrated Nanotechnologies, a U.S. Department of Energy, Office of Basic Energy Sciences user facility. Sandia is a multiprogram laboratory operated by Sandia Corporation, a Lockheed Martin Co., for the U.S. Department of Energy under Contract No. DE-AC04-94AL85000. A portion of this work (LANL) was supported by the U.S. Department of Energy's Office of Electricity Delivery and Energy Reliability.

${ }^{1}$ A. Alu, M. G. Silveirinha, A. Salandrino, and N. Engheta, Phys. Rev. B 75, 155410 (2007).

${ }^{2}$ D. Korobkin, Y. A. Urzhumov, B. Neuner, C. Zorman, Z. Zhang, I. D. Mayergoyz, and G. Shvets, Appl. Phys. A: Mater. Sci. Process. 88, 605 (2007)

${ }^{3}$ R. Ruppin and R. Englman, Rep. Prog. Phys. 33, 149 (1970).

${ }^{4}$ J. R. Aronson and A. G. Emslie, Appl. Opt. 12, 2573 (1973)

${ }^{5}$ J. W. Salisbury and A. Wald, Icarus 96, 121 (1992).

${ }^{6}$ S. K. Andersson and C. G. Ribbing, Phys. Rev. B 49, 11336 (1994).

${ }^{7}$ S. K. Andersson and G. A. Niklasson, J. Phys.: Condens. Matter 7, 8507 (1995)

${ }^{8}$ J. F. Mustard and J. E. Hays, Icarus 125, 145 (1997).

${ }^{9}$ J. T. Luxon, D. J. Montgomery, and R. Summitt, Phys. Rev. 188, 1345 (1969).

${ }^{10}$ M. Kitajima, Crit. Rev. Solid State Mater. Sci. 22, 275 (1997).

${ }^{11}$ S. S. Ng, Z. Hassan, M. R. Hashim, and M. E. Kordesch, Mater. Chem. Phys. 91, 404 (2005).

${ }^{12}$ A. F. Turner, L. Chang, and T. P. Martin, Appl. Opt. 4, 927 (1965).

${ }^{13}$ H. Ogiso, M. Nakada, S. Nakano, and J. Akedo, Nucl. Instrum. Methods Phys. Res. B 257, 545 (2007).

${ }^{14}$ K. J. Hubbard and D. G. Schlom, J. Mater. Res. 11, 2757 (1996).

${ }^{15}$ V. Matias, J. Hanisch, E. J. Rowley, and K. Guth, J. Mater. Res. 24, 125 (2009).

${ }^{16}$ P. Scherrer, Nachr. Ges. Wiss. Goettingen, Math.-Phys. Kl. 2, 96 (1918)

${ }^{17}$ G. K. Williamson and W. H. Hall, Acta Metall. 1, 22 (1953).

${ }^{18}$ P. Y. Yu and M. Cardona, Fundamentals of Semiconductors: Physics and Materials Properties (Springer, Berlin, New York, 1996).

${ }^{19}$ G. Peckham, Proc. Phys. Soc. London 90, 657 (1967).

${ }^{20} \mathrm{~A} \cos ^{2} \theta / \sin \theta$ height error correction was used.

${ }^{21}$ J. R. Jasperse, A. Kahan, J. N. Plendl, and S. S. Mitra, Phys. Rev. 146, 526 (1966).

${ }^{22}$ K. C. Agarwal, B. Daniel, C. Klingshirn, and M. Hetterich, Phys. Rev. B 73, 045211 (2006). 